\title{
Promoting a healthy lifestyle in prison inmates-a window of opportunity
}

$\mathrm{S}$ ubstantial disparities in the prevalence of obesity and overweight, energy intake and the frequency of physical activity exist among prisoner populations worldwide, reveals a new study in The Lancet.

Obesity, inadequate levels of physical activity and a poor diet are risk factors for noncommunicable diseases such as cancer, cardiovascular disease and type 2 diabetes mellitus. The dramatic increase in the prevalence of these diseases worldwide led the WHO to publish a report on global trends in noncommunicable diseases, which coincided with the United Nations meeting on noncommunicable diseases in September 2011. These initiatives draw upon growing awareness of the health and economic costs - at the individual and population levels-associated with the rising prevalence of these diseases, and underscore the need to develop preventive strategies to stop or reverse these trends. Particularly vulnerable populations are those with a low socioeconomic status or living in public or private institutions, such as care facilities or prisons.

"Whilst prison health is a global health issue, it is very much a neglected one," comments study researcher Emma Plugge. "There was a paucity of research in this area, especially in low-income and middleincome countries, despite the fact that on any one day 10 million people worldwide are in prison," she adds. No previous systematic review had focused on the prevalence of adequate diet and physical activity in prisons.

The analysis included data from 31 studies, which included more than 60,000 prisoners in 884 institutions from 15 countries. Case-control studies, case reports and studies with data collected before 1980 were excluded. The researchers used mean BMI and/or categorical BMI values to assess the prevalence of obesity and overweight; mean calorie intake, fat content and sodium content to assess diet quality; and the number of minutes of physical activity per week or the percentage of prisoners with adequate physical activity levels (>150 min per week according to the WHO definition) to assess the prevalence of adequate physical activity. The prevalences of obesity and overweight and adequate physical activity were compared with those of age-matched and sexmatched populations in the same country and the diet data were compared with the daily allowances recommended by the US Food and Nutrition Board.

The study revealed considerable disparities between male and female prisoners. Male prisoners had lower BMI values than age-matched men in the general population in nine of the ten studies (in six countries) analysed. By contrast, the prevalence of obesity was higher among female prisoners than in age-matched women from the general population in the USA and in Australia (although not in the UK). The authors hypothesize that this discrepancy is due in part to an inappropriate diet given to female prisoners, as they observed that the energy intake of the studied women was higher than the recommended daily allowance. Male prisoners in high-income countries, on the other hand, had an appropriate energy intake, whereas the energy intake of male prisoners in lowincome and medium-income countries was lower than recommended.

The researchers also report on substantial disparities in the prevalence of the studied risk factors between countries. In addition to the female-specific differences in obesity prevalence, the study revealed that both male and female prisoners in the UK were less likely to have sufficient levels of physical activity than age-matched and sex-matched individuals in the general population, whereas the situation was reversed in Australia.

Regardless of total energy intake, the quality of the diet consumed by prisoners

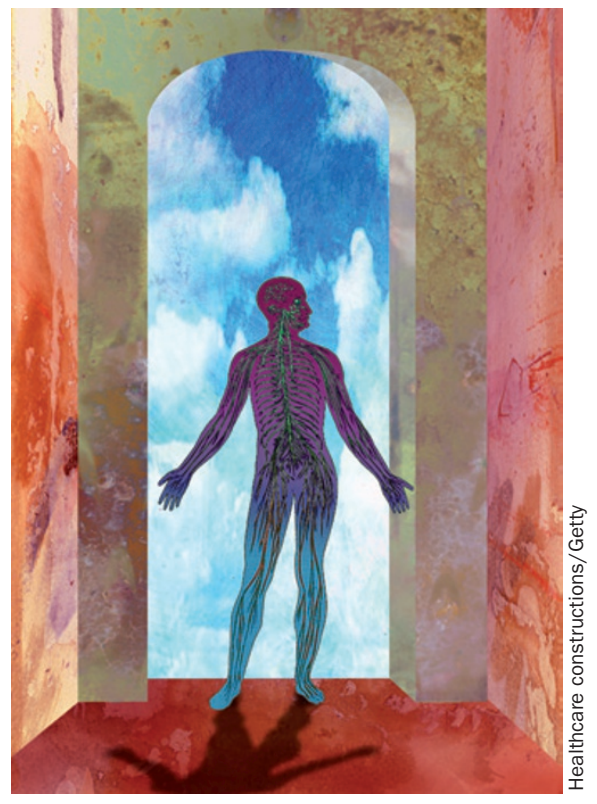

was poor overall, with excessive levels of fat and sodium.

"Further work needs to be done to obtain more basic data on prisoner diets and physical activity worldwide," says Plugge, who also plans to conduct intervention studies in Australia and the UK to examine the health effects of an improved diet and a physical activity programme in prisons.

"Doctors and other health-care staff working in prisons should advise their patients about healthy lifestyles," Plugge points out. "They should lobby the prison authorities to provide a nutritious and balanced diet and adequate opportunities for physical activity," she adds, while noting that prison authorities need to ensure that all prisoners live in humane conditions with access to a healthy diet and adequate physical activity.

Joana Osório

Original article Herbert, K. et al. Prevalence of risk factors for non-communicable diseases in prison populations worldwide: a systematic review. Lancet doi:10.1016/ S0140-6736(12)60319-5 